\title{
Welcome to Acoustics-A New Open Access Journal for the Growing Multidisciplinary Scientific and Engineering Community and Beyond
}

\author{
Jian Kang ${ }^{(1)}$ \\ Founding Editor-in-Chief of Acoustics, UCL Institute for Environmental Design and Engineering, The Bartlett, \\ University College London (UCL), Central House, 14 Upper Woburn Place, London WC1H 0NN, UK; \\ j.kang@ucl.ac.uk; Tel.: +44-(0)20-3108-7338
}

Received: 10 May 2018; Accepted: 15 May 2018; Published: 17 May 2018

Acoustics is a new open access journal, among about 200 diverse, peer-reviewed, open access MDPI journals, supported by over 35,500 academic editors. While the importance of open access has now been widely recognized by authors, readers, and society, as a pioneer in scholarly open access publishing, MDPI has supported academic communities since 1996.

Acoustics is a discipline with significant multi-disciplinary features broadly relating to science, engineering, medical science, social science, and the humanities. It also has a strong practical importance in a range of sectors. Therefore, although there are already a number of acoustics journals in different sectors, there is a recognized need to create an open access journal in acoustics, covering a wide range of topics relating to acoustics, with easy access for all fields and sectors.

The main aim of Acoustics is to deliver rigorously peer-reviewed publications regarding acoustics, and to offer excellent visibility and a fast processing time from submission to publication. It is a multi-disciplinary forum conceived to showcase state-of-the-art research challenges in acoustics science and engineering, and beyond. The topics covered include, but are not restricted to, the following: linear and nonlinear acoustics, physical acoustics, acoustic waves, virtual acoustics, aeroacoustics, infrasound, underwater acoustics, sonar systems, ultrasonics, acoustic materials and metamaterials, acoustical microscopy, engineering acoustics, structural acoustics and vibration, environmental acoustics, soundscape, noise and noise control, building acoustics, room acoustics, duct acoustics, acoustical measurements, computational acoustics, modelling, acoustic signal processing, acoustic sensing, acoustic imaging, hearing, audio, psychological acoustics, speech, voice recognition and control, musical acoustics, and bioacoustics.

Acoustics will publish research articles, systematic reviews, short communications, and perspectives. Moreover, it will publish project reports written by engineers in a structured way. To maximize the advantage of open access, authors are encouraged to include sound files as supplementary materials. Open access journals usually require the author to pay an article processing charge (APC), however, the APC will be fully subsidized by MDPI before 31 December 2019. There are no charges for rejected articles, no submission charges, and no surcharges based on the length of an article, figures, or supplementary data. All accepted papers would go through the in-house layout and English editing process before being returned to the authors for proofreading.

Together with the editorial office (MDPI is based in Basel, Switzerland, and has offices worldwide) and 20+ editorial board members with a strong track record covering a range of topics in acoustics, we will keep publishing high-quality papers continuously. We are also trying to get the journal's papers indexed by key databases including EI, Scopus, ESCI, SCIE, as soon as possible (the current papers may also be indexed retrospectively, depending on the database policies).

We hope to get the journal off to a strong start in its first year by publishing a number of special issues. We welcome your suggestions on appropriate guest editors for each of these topics and 
on other topics that should be explored in the aforementioned special issues. Special issues could also be published in collaboration with conferences. We are also looking for scholars interested in being part of the editorial board of well-known experts, and sharing their extensive expertise and knowledge, with the purpose of building high-standard journals specifically dedicated to their fields of interest. More details on the Editorial Board Member responsibilities can be found at http: / / www.mdpi.com/editors. If you would like to nominate or apply, please send a resume/homepage, along with a list of relevant publications to: acoustics@mdpi.com.

I look forward to receiving your contributions to Acoustics, and welcome your comments and ideas on how to make this an outstanding journal. I can be reached through the Editorial Office (acoustics@mdpi.com) or through my personal email (j.kang@ucl.ac.uk).

(c) 2018 by the author. Licensee MDPI, Basel, Switzerland. This article is an open access article distributed under the terms and conditions of the Creative Commons Attribution (CC BY) license (http:// creativecommons.org/licenses/by/4.0/). 